\title{
Evaluation of Long-Term Potentiation of Small Compound and Unitary EPSPs at the Hippocampal CA3-CA1 Synapse
}

\author{
Michael J. Friedlander, ${ }^{\mathrm{a}}$ Rod J. Sayer, ${ }^{\mathrm{b}}$ and Stephen J. Redman \\ Experimental Neurology Group, John Curtain School of Medical Research, The Australian National University, Canberra, \\ A.C.T. 2601, Australia
}

\begin{abstract}
Long-term potentiation (LTP) was evaluated for small monosynaptic CA3-mediated EPSPs in CA1 neurons in the guinea pig hippocampal slice. Small EPSPs included those elicited by stimulation of Schaffer axon collaterals of several CA3 neurons ( $160-480 \mu \mathrm{V}$ amplitude, $n=40$ EPSPs in 40 neurons) and those elicited by stimulation of an individual CA3 neuron (89-563 $\mu \mathrm{V}$ amplitude, $n=14$ EPSPs in 11 neurons). Various protocols were employed to induce LTP and were deemed successful as evaluated by recording sustained enhancement of the mean peak amplitude of conventionally elicited large compound EPSPs and extracellular field potentials. However, in 47 of 54 cases, tetanization did not lead to a potentiation of the small or unitary EPSPs. In 9 cases, it was possible to directly evaluate the compound EPSP (elicited by stimulating a group of CA3 neuron's axons) and the unitary EPSP (elicited by stimulating a single CA3 neuron) in the same CA1 neuron. The tetanization protocol was successful in inducing LTP in 7 of 9 of these CA1 neurons as evaluated by the compound EPSP but resulted in LTP for only 1 of 9 of the unitary EPSPs for the same neurons. One explanation for these results is a threshold mechanism controlling the expression of LTP. Although LTP induction occurred in most cases, it is proposed that a critical level of depolarization (achieved by the test activation of a sufficient number of CA3 neurons) is necessary so that the enhancement at the modified synapse is expressed.
\end{abstract}

Long-term potentiation (LTP) is a sustained enhancement of synaptic transmission following tetanic activation of the presynaptic neurons. LTP was first described in the dentate gyrus of the rabbit (Bliss and Gardner-Medwin, 1973; Bliss and Lomo, 1973) and since then has been demonstrated at a variety of sites both within the hippocampal formation (see reviews by Teyler and DiScenna, 1984, 1987; Smith, 1987; Gustafsson and Wigstrom, 1988) and in other regions of the CNS (Brown and McAfee, 1982; Lee, 1982; Gerren and Weinberger, 1983; Artola and Singer, 1987).

Our current understanding of the mechanism of LTP induc-

\footnotetext{
Received Feb. 13, 1989; revised Oct. 2, 1989; accepted Oct. 3, 1989.

This research was supported by the John Curtin School of Medical Research (S.J.R.), NH \& MRC of Australia (R.J.S.), and NIH grant EY05116 (M.J.F.) and Fogarty grant TWO-1378 (M.J.F.). We thank Rosemary Enge for word-processing. Correspondence should be addressed to Dr. S. J. Redman at the above address.

" On leave from Neurobiology Rescarch Center, Volker Hall, University of Alabama at Birmingham, Birmingham, AL 35294.

b Present address: Department of Physiology and Biophysics, School of Medicine, University of Washington, Seattle, WA 98195.

Copyright (C) 1990 Society for Neuroscience $0270-6474 / 90 / 030814-12 \$ 02.00 / 0$
}

tion and expression is based largely on analysis of synaptic responses recorded from populations of neurons (field potentials) or from intracellular recordings (see Gustafsson and Wigstrom, 1988, for a review). In both types of experiment, it is the EPSP resulting from the activation of a group of afferents (compound EPSP) that is potentiated. Thus, the properties of LTP that are best documented and particularly attractive as models for learning and memory (Hinton and Anderson, 1981; Teyler and DiScenna, 1984) such as specificity (Dunwiddie and Lynch, 1978; Andersen et al., 1980) and cooperativity (Bliss and Gardner-Medwin, 1973; McNaughton et al, 1978; Yamamoto and Sawada, 1981; Gustafsson and Wigstrom, 1986; Larson and Lynch, 1986; Robinson, 1986) are based on the analysis of compound EPSPs. LTP induction has been demonstrated to depend on postsynaptic depolarization during a tetanus (Douglas et al., 1982; Wigstrom and Gustafsson, 1985; Malinow and Miller, 1986; Sastry et al., 1986; Wigstrom et al., 1986a; Gustafsson et al., 1987) that subsequently releases a $\mathrm{Mg}^{2+}$ block of the synaptically activated NMDA receptor-channel complex (Collingridge et al., 1983; Harris et al., 1984; Wigstrom and Gustafsson, 1985; MacDermott et al., 1986; Wigstrom et al., 1986b), followed by $\mathrm{Ca}^{2+}$ influx. This leads to a series of biochemical changes that modify the biophysical and/or structural properties of the synapse or nearby dendritic spine or membrane in some as yet undetermined way leading to enhancement of subsequent synaptic responses.

The expression of LTP is not associated with any measurable changes in resting membrane potential or input resistance of the postsynaptic neuron (Andersen et al., 1980; Barrionuevo and Brown, 1983); nor is it due to a reduction in synaptic inhibition (Haas and Rose, 1984; Wigstrom and Gustafsson, 1985; Abraham et al., 1987; Taube and Schwartzkroin, 1987). A variety of mechanisms have been suggested to mediate LTP expression including morphological changes at the synapse (Lee et al., 1980; Chang and Greenough, 1984; Andersen, 1989), postsynaptic modification of voltage-sensitive $\mathrm{Ca}^{2+}$ channels (Connor et al., 1988), increased numbers of receptors for the neurotransmitter, glutamate (Baudry et al., 1980; Lynch ct al., 1982; Lynch and Baudry, 1984; but see Lynch et al., 1976; Taube and Schwartzkroin, 1988), and enhancement of release of neurotransmitter from presynaptic terminals (Skrede and Malthe-Sorenssen, 1981; Dolphin et al., 1982; Bliss et al., 1986; Lynch and Bliss, 1986; Errington et al., 1987). All of these mechanisms have features that are compatible with the requirements of specificity and cooperativity such that those inputs that were active during a tetanus are potentiated and concurrent activity of neighboring afferents can strengthen weak inputs.

Changes in synaptic transmission associated with LTP have 


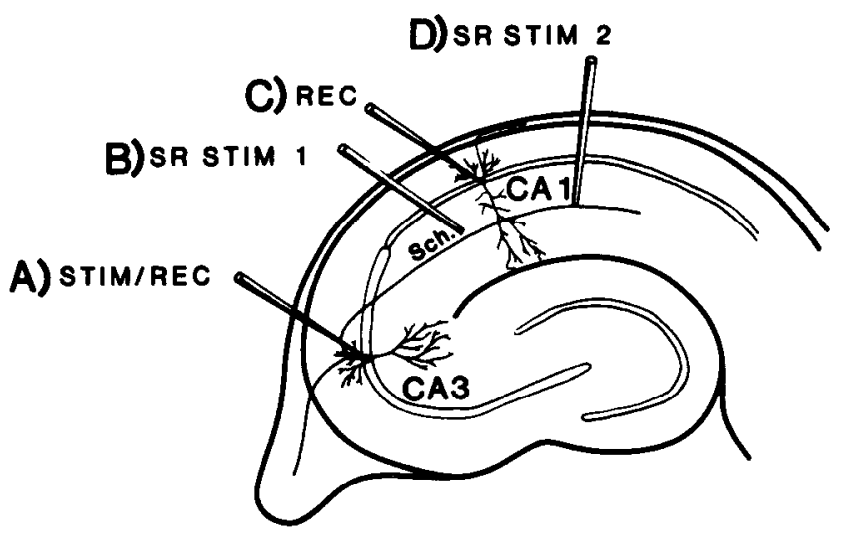

SMALL E.P.S.P.
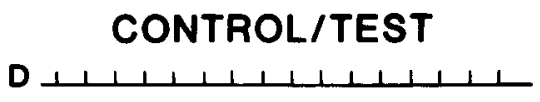

TETANUS PROTOCOL
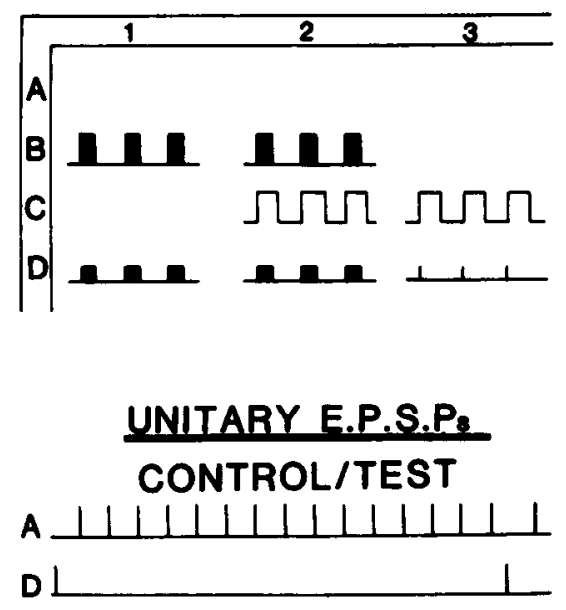

TETANUS PROTOCOL

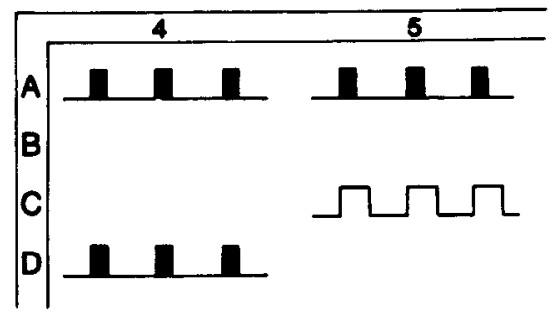

Figure 1. Schematic drawing of hippocampal slice indicating electrode placement for various LTP protocols. Electrode A is a micropipette used to record intracellularly and pass current pulses to directly stimulate a single CA3 neuron. Electrode B is a tungsten stimulating electrode (1) placed in the stratum radiatum (SR) to activate the Schaffer $(S c h$.) axon collaterals of the CA3 neurons running from the CA3 to the CA1 field. Electrode $\mathrm{C}$ is a micropipette used to record intracellularly (and occasionally deliver depolarizing current pulses) from a CAl neuron. Electrode $\mathrm{D}$ is a tungsten stimulating electrode (2) located in the SR distal to the CA1 neuron recording site. Electrode D is used for test stimulation of Schaffer collaterals to elicit compound EPSPs from CA1 neurons, to antidromically activate CA3 neurons, and to deliver tetani to the SR. LTP protocols: The upper and lower panels illustrate the test paradigm and tetanus protocols used to evaluate small compound and unitary EPSPs, respectively. The letters refer to the 4 electrodes $(A-D)$ indicated. not been investigated when only a small number of afferents are activated simultaneously or when a single afferent is activated. As a result of our recently described technique (Sayer et al., 1989,1990 ) for simultaneous intracellular recording from synaptically coupled CA3-CA1 pairs of neurons in the hippocampal slice preparation, it is possible to determine whether LTP can be induced and expressed at unitary connections. Thus, the magnitude of the postsynaptic response required to reveal LTP and the manner in which LTP may be used in subsequent processing in the hippocampus can be evaluated. A preliminary account of some of these findings has appeared (Sayer et al., 1988).

\section{Materials and Methods}

The details of the preparation and maintenance of guinea pig hippocampal slices (Sayer et al., 1989) and the basic electrophysiological procedures for dual intracellular recording from synaptically coupled neuronal pairs in the CA3 and CA1 regions have been presented elsewhere (Sayer et al., 1990).

Simultaneous intracellular recordings were made in an Oslo-type interface chamber from CA3 and CA1 neurons with micropipettes filled with $2 \mathrm{M}$ potassium methylsulfate. One or two insulated $25 \mu \mathrm{m}$ tip diameter tungsten microelectrodes were positioned in the stratum radiatum (SR). Criteria for locating CA3/CA1 neurons, stimulation, recording, digitization, and analysis of intracellular records were as described (Sayer et al., 1990).

Several protocols were used to induce LIP of small or unitary EPSPs evoked at Schaffer axon collaterals with CA1 neurons. These are illustrated schematically (Fig. 1). In all experiments, the same slice was never used to test for LTP in more than one CAl cell since tetani delivered to the SR would probably affect the synapses on other cells in the slice. SR stimulus intensity and frequency were kept to a minimum while searching for cells to avoid premature induction of LTP. Pretetanus records of individual and averaged EPSPs (whether large or small compound EPSPs or unitary EPSPs) were taken in response to single stimuli delivered at $2 \mathrm{~Hz}$ for 5-20 min before tetanization, followed by a 10 $90 \mathrm{sec}$ tetanization period (10-15 sec intertrain interval) during which 1-6 trains consisting of 20 stimulus pulses were delivered at $100 \mathrm{~Hz}$

Small compound EPSPs: The control and test stimuli (delivered preand posttetanus, respectively) consisted of $2.0 \mathrm{~Hz}$ pulses delivered to the SR through electrode D, sufficient to elicit a compound EPSP in the CA1 neuron of $\angle 500 \mu \mathrm{V}$ amplitude during the control period. Three tetanus protocols were used to evaluate LTP for small compound EPSPs. The solid rectangles indicate a train of 20 pulses of 20-100 nA delivered at $100 \mathrm{~Hz}$ through the electrodes indicated. The open rectangles indicate depolarizing pulses applied directly through the intracellular recording microelectrode $(C)$ to the $C A 1$ neuron. These pulses were 5-7 nA and $100-400 \mathrm{msec}$ in duration timed for onset at the peak of the small compound EPSP. The individual pulses indicated in protocol 3 for electrode $D$ indicate single pulses applied at the test rate and strength to the SR but conjoined with direct depolarization of the CAl neuron. Unitary EPSPS: The control and test stimuli (pre- and posttetanus) consist of (a) $2.0 \mathrm{~Hz}$ intracellular stimulation of the CA3 neuron (electrode A) with a $0.5-5.0 \mathrm{nA}, 3.0-5.0 \mathrm{msec}$ current pulse adjusted to evoke a single action potential from the $\mathrm{CA} 3$ neuron and elicit the unitary EPSP in the CA1 neuron and (b) $0.07 \mathrm{~Hz}$ stimulation (electrode D) of the SR, interleaved with the stimulation through electrode A to elicit the compound EPSP in the same CAl neuron. Two tetanus protocols ( 4 and 5) were employed for evaluation of LTP at synaptic contacts between pairs of individual CA3 and CA1 neurons. Protocol 4 consisted of tetanization of the SR (electrode D; 3-6 trains of 20 pulses each separated by 10-15 sec delivered at current intensity of 9-160 nA) in combination with tetanic activation of the $\mathrm{CA} 3$ neuron being recorded (electrode A) with the same paradigm but using intracellular current pulses of 4-9 nA, sufficient to elicit a single action potential for each pulse. In protocol 5 , the tetanization was applied directly to the individual CA3 neuron only and conjoined with intracellularly applied depolarizing current pulses (7-9 nA, $300 \mathrm{msec}$ ) to the CA1 neuron (electrode $\mathrm{C}$ ). 


\section{A Population Spike}

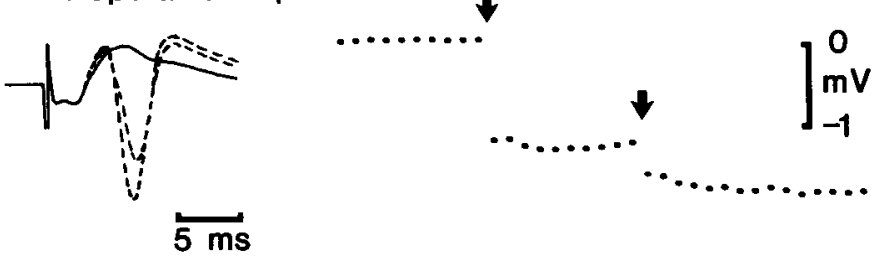

B Intracellular EPSP
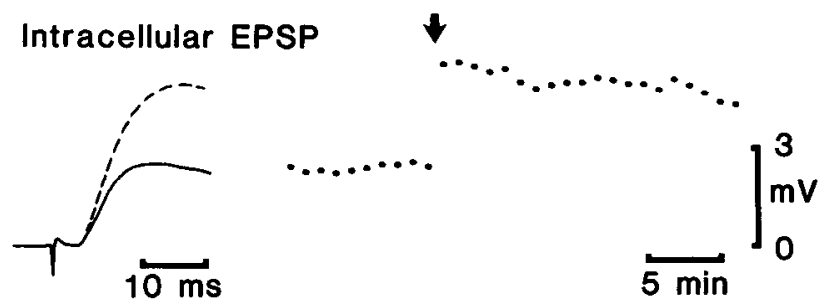

Figure 2. Examples of LTP as evaluated by $(A)$ extracellular recording of field potentials in the CAl field (with concurrent population spike) and $(B)$ intracellularly recorded compound EPSPs from an individual CAl neuron. Control and test stimuli were delivered to the SR (electrode D) at $2.0 \mathrm{~Hz}$. Averaged responses (>120 trials) are shown with the control (pretetanus) records indicated by the solid lines and the test (posttetanus) records indicated by the dashed lines. The tetanus consisted of 3 trains delivered through electrodes B and D (9.8 and 21.0 $\mu \mathrm{A}$, respectively). Two tetani were given for the field potential recording $(A)$. Note the marked enhancement of the slope of the field EPSP and the appearance of the population spike. The second tetanus resulted in further enbancement of the field potential and population spike. The minute average of the population spike is shown to the right. The arrows indicate tetanization (as they do in all subsequent figures). Note the sustained enhancement of the response after tetanization. A single tetanus was delivered $(B)$ for the intracellular recording of the compound EPSP. Note the marked enhancement of the amplitude of the compound EPSP and the sustained effect as indicated by the minute average.

followed by a test period of 15-180 min during which posttetanus records of individual responses and averaged EPSPs were taken at $2 \mathrm{~Hz}$.

The overall quality of the intracellular recordings and the resting membrane properties (resting potential, input resistance as evaluated in discontinuous current mode and action potential threshold and amplitude) were evaluated for each CAl neuron during pre- and posttetanus periods.

Small compound EPSPs. Protocols 1, 2, and 3 (Fig. 1) were used for evaluating LTP for 40 small $(<500 \mu \mathrm{V}$ mean peak amplitude) compound EPSPs. In protocol $1(n=26)$, cells were tetanized through the test electrode at the test stimulus strength, with a concurrent stronger (20$100 \mu \mathrm{A})$ but identically timed tetanus delivered through electrode $\mathrm{B}$ placed equidistant from the stratum pyramidale. This allowed afferents projecting to the same regions of the CAl cell's apical dendrites to be activated by both electrodes. The advantage of this protocol was that the test stimulus (delivered from electrode D in Fig. 1) did not have to be adjusted for delivery of a strong tetanus (with the possibility of hysteresis in the constant current stimulus isolator controls on resetting it) because the strong synaptic depolarization was provided by the other input (electrode B in Fig. 1). It also eliminated concerns about local tissue damage caused by the greater currents during the tetani which might have affected the test EPSP.

Protocol 2 consisted of the same tetanization applied to the 2 SR stimulating electrodes (B and D) with additional depolarization provided directly to the CA I neuron via intracellular current injection (200$300 \mathrm{msec}$ pulses of 5-10 nA applied through electrode $C$ so that pulse onset coincided with tetanus onset). This protocol assured that a direct depolarization of the CAI neuron coincided with the activation of a small group of axons near electrode $D$ in the unlikely event that the axons of the SR that were tetanized by electrode $B$ had their input on a part of the dendritic arbor of the CAl neuron that was electrotonically isolated from the inputs tested by electrode $D$.
Protocol 3 called for conjoining depolarizing current pulses (intracellular current injection into the CAl neuron via electrode $C$ ) with the test stimulus pulses applied at $2 \mathrm{~Hz}$ through electrode $\mathrm{D}$. The conjunction period lasted 3-6 min. The depolarizing pulses (5-7 nA, 100-400 msec) were timed to start at the peak of EPSP as previously described (Wigstrom et al., 1986a; Gustafsson et al., 1987). No tetanus was applied during protocol 3 . Such conjunctions have been previously reported to potentiate large compound EPSPs (Gustafsson et al., 1987) by more than $70 \%$

In the experiments with small compound EPSPs, $10 \mu \mathrm{M}$ bicuculline methiodide was added to the ACSF to reduce $\mathrm{GABA}_{\mathrm{A}}$-mediated synaptic inhibition. This was done to reduce contamination by IPSPs and to facilitate the induction of LTP (Wigstrom and Gustafsson, 1985). Also, CA3 was surgically removed to prevent propagation of spontaneous activity from the disinhibited $\mathrm{CA} 3$ region.

Unitary EPSPS. Two protocols were used to evaluate LTP for unitary EPSPs for single CA3-CA1 cell pairs. Protocol 4 consisted of applying tetani to the SR (electrode D) in conjunction with direct intracellular tetanic stimulation of the CA3 cell under study (electrode A). This assured that even if the tetanic activation of the Schaffer collaterals was below threshold for activating the axon of the CA3 neuron under study (but suprathreshold for activating neighboring axons), the CA3 cell would be fired repetitively by direct application of current pulses. Thus, the criteria for cooperativity by synaptic activation of the CAl neuron via the specific CA3 neuron under study and the neighboring axons was met. The current strength of the stimulus train applied directly to the CA3 cell via intracellular current injection (electrode A) was adjusted above test levels to be certain that an action potential was elicited in the CA3 neuron for each pulse. This was also verified by collecting records from the CA3 neuron during application of the tetanus. The current strength of the tetanus applied to the SR via electrode $D$ was independently set through a second stimulus isolation unit linked to the first unit with a switch.

Control (pretetanus) and test (posttetanus) records were obtained by recording the compound and unitary EPSPs in the CA1 neuron elicited by SR stimulation (electrode D) and single CA3 cell stimulation (electrode A), respectively. These stimuli were presented in an interleaved fashion with activation of electrode $A$ at $2 \mathrm{~Hz}$ and activation of electrode D every 15 sec.

Protocol 5 involved tetanizing only the single CA3 cell (electrode A) in conjunction with a direct depolarization applied via intracellular current injection (electrode $\mathrm{C}$ ). The rationale for this protocol was to evaluate whether LTP could be induced if appropriate postsynaptic depolarization occurred (via electrode $\mathrm{C}$ ) while only a single CA3-CA1 connection was activated (via electrode A). This protocol prohibited cooperative interactions between presynaptic terminals from ncighboring afferents.

Analysis and controls. The large compound EPSPs, small compound EPSPs, and unitary EPSPs were evaluated by comparing the average of the peak amplitude, taken minute by minute, and the averaged EPSP for 5-10 min epochs of the pre- and posttetanus periods. Individual records were also compared by constructing amplitude distribution histograms to search for evidence of changes in quantal size and release probabilities (see Sayer et al., 1989, 1990).

Control experiments were done to verify that LTP could be routinely generated in the slices as evaluated by conventional criteria including extracellular recordings of field potentials and intracellular recording of conventional large (1.0-2.5 mV) compound EPSPs evoked at the same frequency as for the unitary EPSPs $(2 \mathrm{~Hz})$. Moreover, recordings of unitary EPSPs were made from CA3-CAl pairs to demonstrate another form of synaptic plasticity for unitary contacts - paired-pulse facilitation. While recording from a synaptically coupled pair of neurons, short current pulses were delivered to the CA3 neuron, adjusted to evoke single action potentials, and subsequently delivered in pairs separated by $15-200 \mathrm{msec}$. A range of paired-pulse intervals (15-200 msec) was tested although most pairs were only tested at $25 \mathrm{msec}$ intervals. Averages were constructed off-line from the 200-300 records saved for each interval.

The peak of the second EPSP of each pair usually occurred during the decay phase of the first EPSP. Therefore, the voltage contributed by the first EPSP to the peak amplitude of the second EPSP was calculated and subtracted, before determining the degree of facilitation. The degree of facilitation of the second EPSP was then expressed as the percentage increase of its mean peak amplitude over that of the first EPSP. 


\section{Results}

Complete analysis of one of the LTP protocols was carried out for 68 cases of intracellular recording from CAl neurons. (The number of CAl neurons from which these records were obtained was slightly less since more than one protocol was applied to the same cell in several cases.) The data base consists of LTP of large (1.2-2.5 mV) compound EPSPs $(n=4)$; LTP of small $(<500 \mu \mathrm{V})$ compound EPSPs by protocol $1(n=26)$, protocol $2(n=9)$, protocol $3(n=5)$; LTP of unitary EPSPs by protocol $4(n=9)$, protocol $5(n=5)$; paired pulse facilitation of unitary EPSPs $(n=10)$. In addition, LTP was evaluated by extracellular recordings of field potentials and population spikes in 4 cases. The results of the LTP experiments are presented in ascending order with respect to resolution and spatial minimization of the tetanus. Thus, the first results considered are the extracellular recording of field potentials, followed by the large compound EPSPs, followed by the small compound EPSPs, followed by the unitary EPSPs during SR tetanization, followed by the unitary EPSPs during tetanization of a single neuron. The results of the experiments of paired-pulse facilitation at unitary contacts are considered last.

\section{Field potentials and large compound EPSPS}

An example of the potentiation of the field potential and population spike in the CA1 region after application of 2 sets of 3 train tetani is shown in Figure $2 A$. The averaged field potential is indicated, and the potentiated responses after tetani 1 and 2 are shown. There was no obvious population spike before the tetanus. The other 3 field potentials that were evaluated for LTP showed similar persistent increases in the population spike amplitude after tetanic activation.

Protocols for inducing LTP were tested on 4 intracellularly recorded compound EPSPs with peak amplitudes in the range 1.5-2.5 $\mathrm{mV}$ during the control period. Three of these EPSPS showed a sustained potentiation after the tetanus delivered to the 2 stimulating electrodes in the SR. One result is illustrated in Figure $2 B$, where the peak amplitude increased by $100 \%$ during the period 5-10 min after the tetanus. The other 3 large compound EPSPs increased by $90 \%, 39 \%$, and one ( $4 \%$ increase) did not change after tetanization. These experiments indicate that our standard protocols for tetanizing the SR were reliable for inducing LTP throughout the CAl region as evaluated by recording field potentials and by conventional intracellular recordings of compound EPSPs from individual CA1 cells in most cases.

\section{Small compound EPSPS}

The small compound EPSPs evoked by stimulation of the SR were investigated using protocols 1-3. Representative examples of the minute averages of the peak amplitude of these small EPSPs before and after these various tetanus protocols are illustrated in Figure 3. Figure 3, $A-C$ shows examples from the largest group tested (protocol $1, n=26$ ). The arrows indicate the timing of the tetanus, which in each case consisted of 3 trains (strength of the tetani are indicated in figure legend). The minute averages illustrated in Figure $3, A$ and $B$, and are representative of the response of most ( 24 of 26) of the small compound EPSPs tested with this protocol. Their amplitude did not change after tetanization. The mean individual percentage change in amplitude from the control period to the 5-10 min posttetanus period was $0.5 \%(\operatorname{SEM}=6.1 \%)$. The mean amplitudes (for the 24

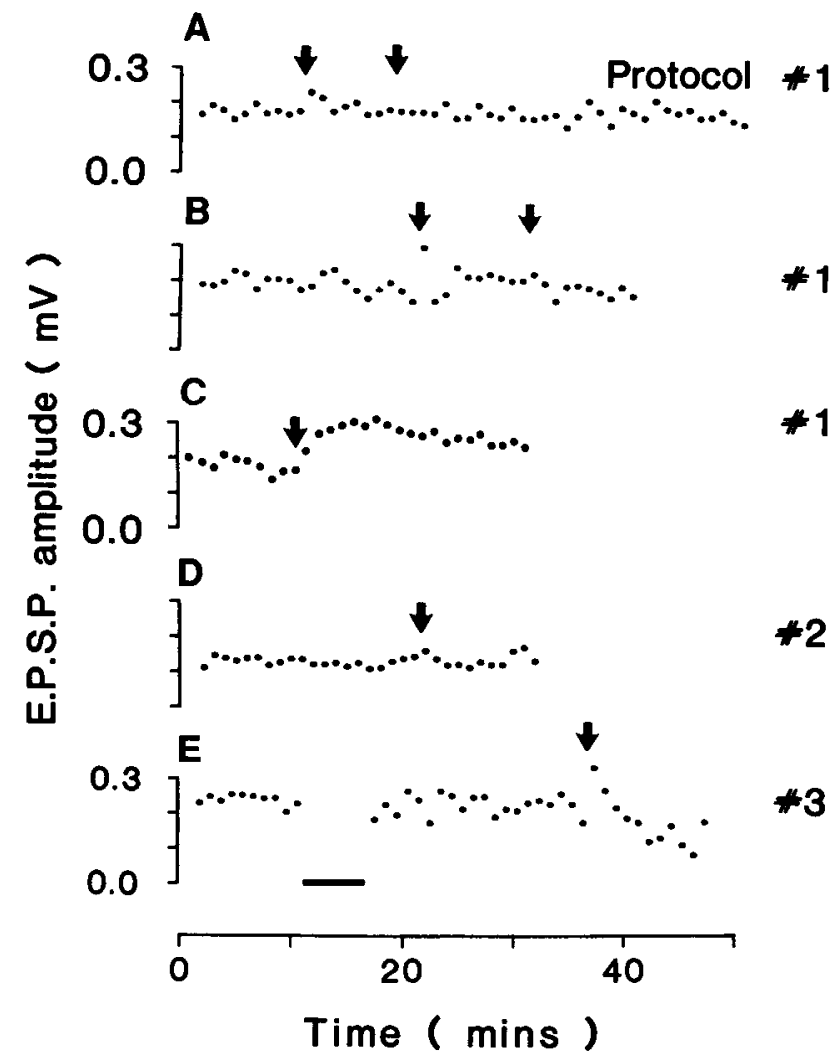

Figure 3. Minute averages for a representative sample of 5 of the small compound EPSPs that were evaluated with tetanus protocols $1-3$ ( $n=$ 40). The protocols used with each EPSP are indicated. The largest sample ( $n=26$ ) was evaluated with protocol $1 . A-C$. Three examples of minute averages before and after tetanization with protocol 1 . The mean peak amplitude of the small EPSPs illustrated in $A$ and $B$ did not increase after the tetanus (except for a transient posttetanic potentiation lasting less than $1 \mathrm{~min}$ ). These examples are indicative of the response of most (24 of 26) of the small compound EPSPs. The example illustrated in $C$ is 1 of 2 cases where the minute average demonstrated a sustained potentiation (although even in this best case, the mean peak amplitude of the small compound EPSP had returned nearly to control levels 20 min posttetanus). The tetani consisted of trains of 20 pulses delivered at $100 \mathrm{~Hz}$ through electrodes $\mathrm{B}$ and $\mathrm{D}$. The specific tetanus parameters were as follows: $A, 3$ trains for each tetani, $3.4 \mu \mathrm{A}$ through electrode $\mathrm{D}$ 44 and $60 \mu \mathrm{A}$ through electrode $\mathrm{B}$ for tetani 1 and 2 , respectively; $B, 3$ trains for each tetanus, $2.7 \mu \mathrm{A}$ through electrode $\mathrm{D}$ and $200 \mu \mathrm{A}$ through electrode $B$ in each case; $C, 3$ trains, 18 and $25 \mu \mathrm{A}$ through electrodes $\mathrm{D}$ and $\mathrm{B}$, respectively. $D$, Example of minute average with protocol 2 . The tetanus consisted of 3 trains, 3 and $50 \mu \mathrm{A}$ through electrodes D and $B$, respectively, conjoined with $10 \mathrm{nA}, 300 \mathrm{msec}$ depolarizing pulses delivered through electrode C. $E$, Example of minute average with protocol 3. The bar indicates a 6 min conjunction of the test stimulus ( 8 $\mu \mathrm{A}$ through electrode $\mathrm{D}$ continued at $2.0 \mathrm{~Hz}$ ) with $7 \mathrm{nA}, 400 \mathrm{msec}$ depolarizing pulses injected through electrode $\mathrm{C}$. The arrow indicates an additional attempt to induce LTP of the small compound EPSP via tetanization (at $80 \mu \mathrm{A}, 3$ trains) through electrode $\mathrm{B}$.

EPSPs) were 223 and $226 \mu \mathrm{V}$ before and after the tetanus, respectively ( $1.4 \%$ increase). A small transient increase in the amplitude of the small compound EPSP persisting 1-2 min (note the point immediately after the first tetanus in records illustrated in Fig. 3, $A, B$ ) was common before the rapid return of the amplitude to baseline. These results were consistent for the 24 small EPSPs even after multiple tetani at higher intensities. (Note the 2 periods of tetanization for the record in Fig. 3, $A$, B.) 


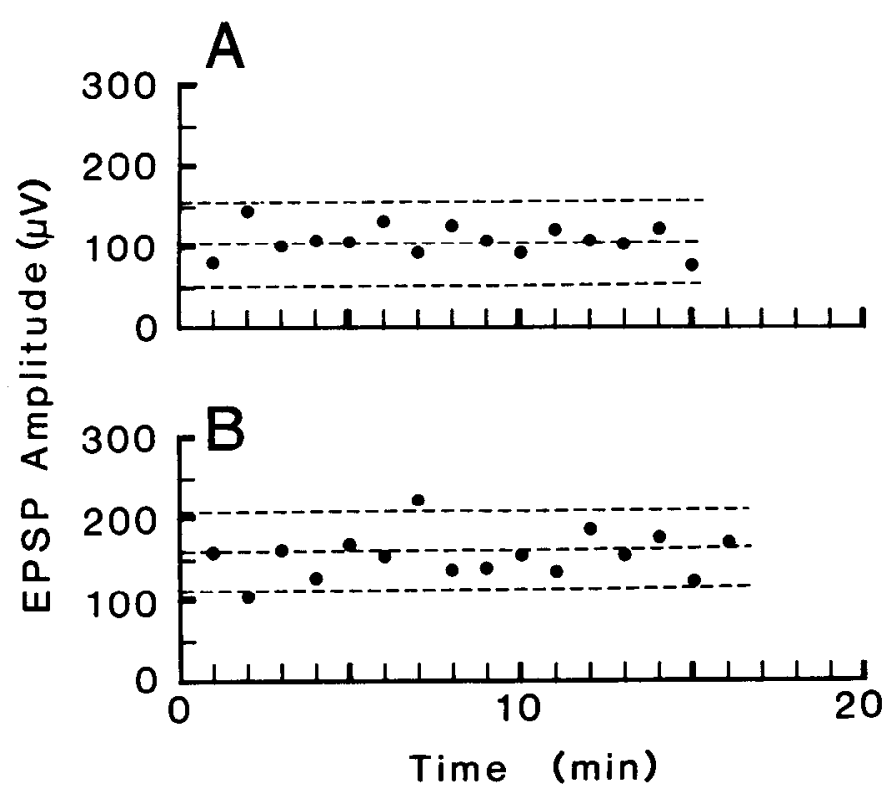

Figure 4. Minute averages of 2 unitary EPSPs for illustration of stability of mean peak amplitude during recording period. Each point is the average of 120 records taken at $2.0 \mathrm{~Hz}$. The inner dashed lines indicate the overall mean peak amplitude over the recording period. The outer dashed lines indicate the $95 \%$ confidence intervals. The minute averages illustrated in $A$ and $B$ are typical of most unitary EPSPs (20 of 23 tested that were not subjected to any other protocols such as tetanization) in that they were very stable over time.

For 2 of 26 cases, the small EPSPs did show an increased peak amplitude in the minute averages ( 88 and $84 \%$ increase initially) that persisted beyond the first minute posttetanus. One of these is illustrated in Figure $3 C$. However, even in this rare case of potentiation of a small $(200 \mu \mathrm{V})$ compound EPSP, the peak amplitude had nearly returned to control levels within 15 min after the tetani.

Protocol 2 was applied to $9 \mathrm{CAl}$ cells. An example of the minute averages of the peak amplitude for one of the small compound EPSPs is illustrated in Figure 3D. Even though the SR tetanus was conjoined with a strong (10 nA) direct depolarization of the CA1 neuron via intracellular current injection, the small $(125 \mu \mathrm{V})$ compound EPSP did not change in amplitude after the tetani. This response was indicative of the 8 of 9 neurons tested with protocol 2 . The overall mean amplitude of the small compound EPSPs for this group showed a slight decline (-7.0\%) from $126 \mu \mathrm{V}$ to $117 \mu \mathrm{V}$ after the tetani conjoined with depolarizing pulses of varying strengths (8-15 $\mathrm{nA}, 300 \mathrm{msec}$ duration conjoined with each train in a tetanus). The ninth small compound EPSP showed a gradual incrcase in amplitude (by $41 \%$ over $10 \mathrm{~min}$ ) but returned to baseline within $30 \mathrm{~min}$.

Five small compound EPSPs were tested with protocol 3 . The afferent fibers were not tetanized but received continuous lowfrequency stimulation of the SR at the standard test rate $(2 \mathrm{~Hz})$ in conjunction with direct depolarization (via intracellular current injection). An example of the minute average of the peak EPSP amplitude for one of these cells is indicated in Figure $3 E$. The bar indicates the 6 min conjunction period. The amplitude did not increase. This cell received an additional tetanization via protocol 1 at the time indicated by the arrow. After a transient (1-2 $\mathrm{min}$ ) increase, the amplitude declined to below control levels. Three of the 5 EPSPs tested by protocol 3 showed min- imal change (1-12\% declines) in the peak amplitude. The remaining 2 EPSPs showed transient increases in amplitude (59 and $33 \%$ ) after the conjunction, but neither remained consistently elevated.

In summary, the small compound EPSPs in 35 of 40 cells tested by a variety of protocols showed no signs of LTP. The other EPSPs showed a variety of variable and short-lived enhancements. This result obtained despite repeated tetanizations in many cases. Moreover, similar protocols induced LTP as evaluated by recording extracellular field and conventional "large" compound EPSPs in these same experimental preparations.

\section{Unitary EPSPS}

In order to evaluate LTP for unitary EPSPs, it was first necessary to determine the degree of variability in response amplitudes over the periods of time sampled. While the trial-to-trial variability, reflecting both the background noise and intrinsic fluctuations in the amplitude of the EPSP (Sayer et al., 1989, 1990), has been described, the minute-by-minute average of the unitary EPSP amplitudes has not. The minute averages of a sample of unitary EPSPs that were not experimentally manipulated (no attempts at LTP induction) were evaluated for trends and variability in the mean peak amplitudes. Examples of this analysis are illustrated in Figure 4. The averages in Figure 4, $A$ and $B$, are indicative of 20 of 23 unitary EPSPs analyzed in this fashion. They are stable and show no trends in amplitude. Virtually all of the points fall within the $95 \%$ confidence intervals of the mean for the whole sample of the unitary EPSP as expected for a stationary condition.

Unitary EPSPs from 9 CA1 neurons were successfully carried through protocol 4 . In $7 \mathrm{CA} 1$ cells, the tetani produced sustained enhancement in the amplitude of the compound EPSP. An example is illustrated in Figure 5, and it demonstrates a clear prolonged enhancement of the amplitude of the compound EPSP following the tetanus, with no corresponding change in the unitary EPSP (Fig. $5 B$ ). The records obtained during the 3 tetanic trains delivered to this cell are shown in Figure $5 C$. The trains of current pulses injected into the CA3 cell elicited action potentials at the appropriate frequency. Except for the first pulse in each train, which discharged the CA3 cell twice, there is a single action potential on each pulse. The CA1 records illustrate the repetitive discharge superimposed on a slow depolarization as a result of the tetanus delivered through the stimulating electrode (each stimulus was $80 \mu \mathrm{A}, 0.1 \mathrm{msec}$ duration). Averages of the compound and unitary EPSPs are shown in Figure $5 \mathrm{~A}$, with the pre- and posttetanus averages superimposed. The compound EPSPs remained larger than their control amplitude for the duration of the recording (17-90 min posttetanization) for these cells.

Table 1 lists the mean amplitudes of both the compound and unitary EPSPs in the 9 cells for the 5 min prior to the tetani and for the 5-10 min period following the tetani. The increase in the peak amplitude for the 7 compound EPSPs that were potentiated ranged from 37 to $266 \%$ (mean $=84 \%$, SEM $=24 \%$, $n=7$ ). In contrast, over the same interval, 6 of the 7 unitary EPSPs did not increase in amplitude ( 3 unitary EPSPs declined by $22-26 \%$ and 3 showed minimal change: $0-5 \%$ ). Within the group of 7 CA1 neurons for which LTP occurred (as evaluated by $>35 \%$ enhancement of the compound EPSP), only one of the unitary EPSPs increased in amplitude (32\%), but even this unitary EPSP declined to control levels within $15 \mathrm{~min}$. At that 


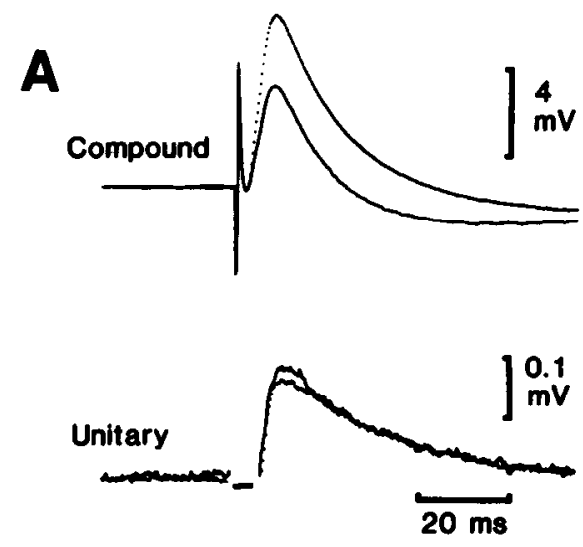

C
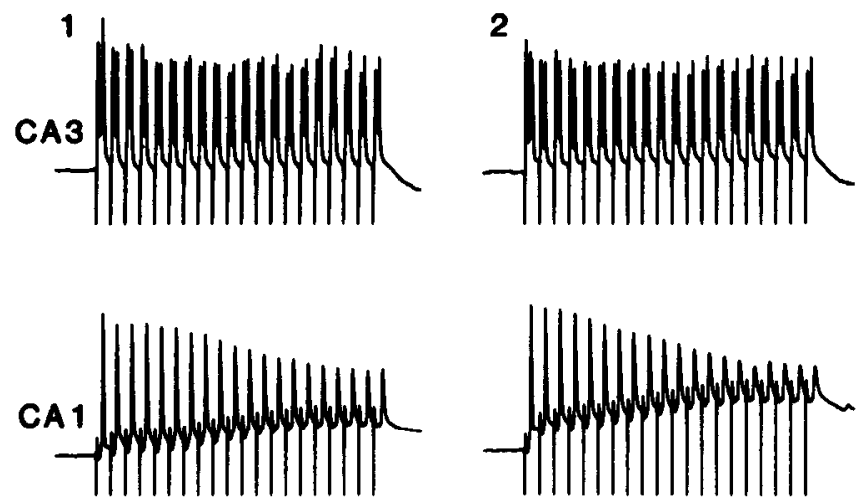

Figure 5. Avcrages $(A)$ and minute averages $(B)$ of compound and unitary EPSPs in the same CAl neuron before and after tetanization with protocol 4 . $A$, Averages of the compound EPSP evoked by a $40 \mu \mathrm{A}$ stimulus (at 0.07 $\mathrm{Hz}$ ) through electrode D and the unitary EPSP evoked by depolarizing current pulses (delivered at $2.0 \mathrm{~Hz}$, each pulse eliciting a single action potential in the CA3 neuron) through electrode A. Solid lines are pretetanus averages, and dotted lines are posttetanus averages. $B$, Minute average records of the mean pcak amplitudes of the compound and unitary EPSPs recorded from the same CA1 neuron illustrated in $A$. The tetanus consisted of 3 trains, delivered through electrode D $(80 \mu \mathrm{A}$ strength) and through electrode A. $C$, Responses of the CA3 and CA1 neurons during the 3 train tetanus delivered to the SR and the CA3 neuron directly. Note that the $\mathrm{CA} 3$ neuron fired an action potential on each pulse within each of the 3 trains of the tetanus and that the CAl neuron fired with each stimulus also. The membrane potential of the CA1 neuron also became gradually and progressively more depolarized during each train. time the corresponding compound EPSP for the same cell was still more than $200 \%$ of its control amplitude.

When considered together, the overall mean amplitudes of the 7 compound EPSPs that were potentiated were 5.3 and 8.4 $\mathrm{mV}$ for the pre- and posttetanus periods, respectively (59\% increase). The overall mean amplitudes for the unitary EPSPs in the same neurons for the pre- and posttetanus periods were 277 and $272 \mu \mathrm{V}$, respectively ( $2 \%$ decline). No changes in the resting membrane potential, input resistance, or action potential amplitude occurred after the tetanization.

In summary, the results of tetanization of the SR on the amplitude of unitary EPSPs suggest that LTP is rare, occurring in 0 of 9 cases tested (of which 7 showed clear LTP as evaluated by the compound EPSP).

\section{Tetanization of unitary afferents}

Protocol 5 was used to determine whether activation of a single afferent could induce LTP. In 5 cases, only the individual CA3 afferent that elicited a unitary EPSP in a CA1 neuron was tetanized in conjunction with direct depolarization of the CA1 neuron by intracellular injection. In 4 of the 5 cases, the amplitude of the unitary EPSP showed little or no effect after the tetanization. The minute averages from 3 of these cells are illustrated in Figure $6, A-C$. Figure $6 D$ illustrates intracellular records of the responses of a $\mathrm{CA} 3 / \mathrm{CA} 1$ pair during one of the tetani. Note that the CA3 cell fired regularly on each cycle of the stimulus train and the CA1 cell was strongly depolarized, leading to a sustained burst during the same period.
Table 1. Effects of tetanization on compound and unitary EPSPs evoked in the same CA1 neurons

\begin{tabular}{|c|c|c|c|c|c|c|}
\hline \multirow[b]{2}{*}{ Exp. } & \multicolumn{3}{|c|}{ Compound EPSPs } & \multicolumn{3}{|c|}{ Unitary EPSPs } \\
\hline & Pre & Post & $\begin{array}{l}\text { Change } \\
(\%)\end{array}$ & Pre & Post & $\begin{array}{l}\text { Change } \\
(\%)\end{array}$ \\
\hline 1 & 7.54 & 10.29 & +37 & 0.563 & 0.438 & -22 \\
\hline 2 & 1.41 & 5.15 & +266 & 0.187 & 0.246 & $+32^{a}$ \\
\hline 3 & 4.33 & 7.13 & +65 & 0.143 & 0.143 & 0 \\
\hline 4 & 6.67 & 9.53 & +43 & 0.506 & 0.451 & -11 \\
\hline 5 & 6.42 & 11.23 & +75 & 0.089 & 0.066 & -26 \\
\hline 6 & 8.34 & 12.69 & +52 & 0.336 & 0.338 & +1 \\
\hline 7 & 2.04 & 3.06 & +50 & 0.115 & 0.121 & +5 \\
\hline 8 & 4.51 & 5.40 & +20 & 0.106 & 0.112 & $+6^{h}$ \\
\hline 9 & 1.64 & 1.60 & -2 & 0.397 & 0.386 & $-3 c$ \\
\hline Mean & 4.77 & 7.34 & +67 & 0.271 & 0.256 & -2 \\
\hline
\end{tabular}

Mean peak amplitudes (in $\mathrm{mV}$ ) are listed for the $5 \mathrm{~min}$ period before tetanization (Pre) and the 5-10 min period afterwards (Post).

${ }^{a}$ The neuron from experiment 2 showed an increase in the amplitude of its unitary EPSP after tetanization, but the amplitude returned to near control levels within 15 min while the compound EPSP amplitude remained elevated.

${ }^{b}$ Although the compound EPSP amplitude increased by $20 \%$ in the period immediately following the tetanization, its amplitude returned to near control levels $10-15$ min posttetanus.

c This is the only compound EPSP ( 1 of 9) for the group of CA1 neurons in which both compound and unitary EPSPs were tested where the tetanization protocol did not result in any discernible change in peak amplitude. 


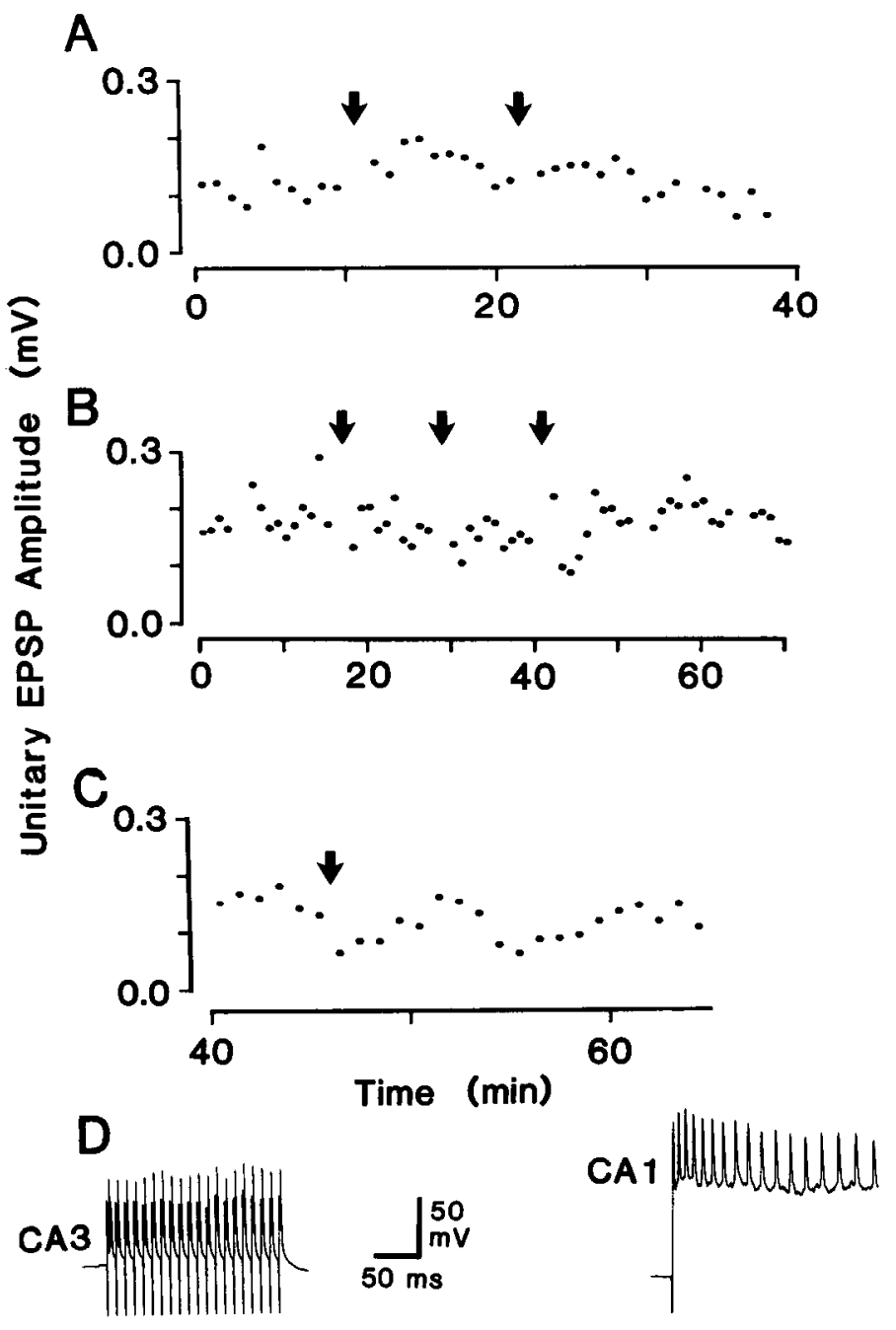

Figure 6. A, Minute averages of unitary EPSPs from 3 CA1 neurons before and after tetanization with protocol 5 . In each case, the tetani were delivered only to the CA3 neuron through electrode $\mathrm{A}$ and conjoined with direct depolarization (through electrode C) of the CAl neuron. Tetanization parameters were as follows: $A, 5$ trains in conjunction with $7 \mathrm{nA}, 300 \mathrm{msec}$ depolarizing pulses for tetanus 1 and $8 \mathrm{nA}, 500$ msec depolarizing pulses for tetanus $2 ; B, 5$ trains conjoined with 7 and $9 \mathrm{nA}, 300 \mathrm{msec}$ depolarizing pulses for tetani 1 and 2 , respectively; 10 trains conjoined with $9 \mathrm{nA}, 500 \mathrm{msec}$ depolarizing pulses for tetanus 3; $C, 3$ trains each conjoined with a $7 \mathrm{nA}, 300 \mathrm{msec}$ depolarizing current pulse. $D$, Responses of the $\mathrm{CA} 3$ and $C A 1$ ncuron (whose minute averages are illustrated in $C$ ) during tetanization protocol 5 . Note that an action potential was generated by the CA3 neuron for each pulse and that the CA1 neuron was tonically depolarized during the application of the intracellular current pulse.

In contrast, a fifth CAl neuron tested with protocol 5 did show a marked potentiation of the amplitude of its unitary EPSP after tetanization of a single CA3 afferent in conjunction with postsynaptic depolarization. The averaged unitary EPSP and minute average of the peak amplitude for this unitary EPSP are illustrated in Figure 7. The arrows (Fig. $7 B$ ) indicate the timing of 2 tetani paired with a postsynaptic depolarizing pulse.

EPSP amplitude histograms (see Sayer et al., 1989, 1990) were constructed from the individual records for the pre- and 2 posttetanus periods for the CAl neuron described above. From these histograms it was apparent that the amplitudes of the posttetanus responses extended over a wider range than the amplitudes

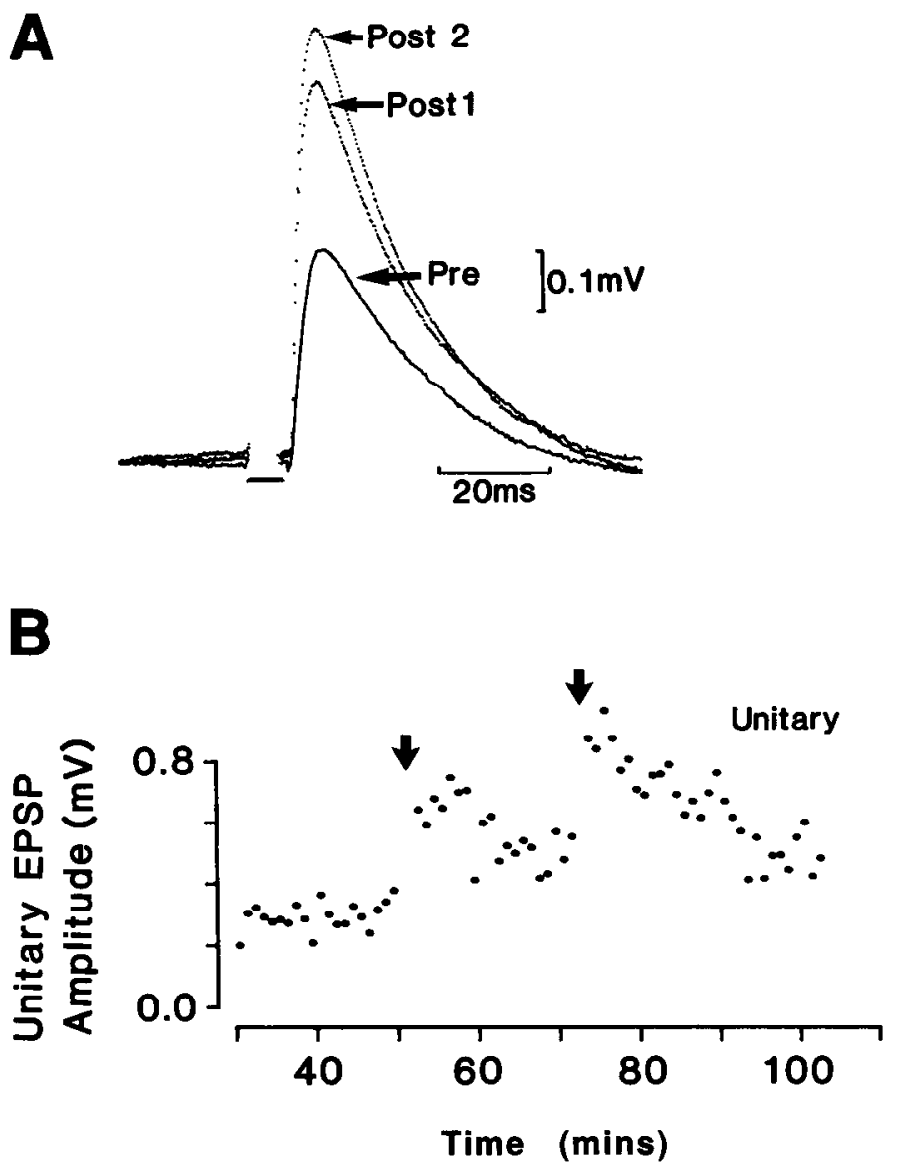

Figure 7. Averages and minute average of a unitary EPSP that showed LTP after tetanization with protocol 5. Each tetanus consists of 6 trains of repetitive activation of the CA3 neuron (electrode $A$ ) in conjunction with $7 \mathrm{nA}, 300 \mathrm{msec}$ depolarizing pulses to the CA1 neuron (electrode C). $A$, Average records of unitary EPSP for the $10 \mathrm{~min}$ period before the tetanus (solid line, Pre) and for comparable periods immediately after the first (dotted line, Post 1 ) and second (dotted line, Post 2) tetanus. Note the marked enhancement of the EPSP after the first tetanus and subsequent further increase after the second tetanus. $B$, Minute averages showing large (but decaying) potentiation of the unitary EPSP amplitude after tetanization of the individual CA3 neuron.

of the control responses, while the SD of the background noise did not alter in these different periods. However, when the deconvolution procedure was applied to the histograms, the average separation between discrete amplitudes in the calculated results was too small with respect to the SD of the noise (see Sayer et al., 1989) for us to have confidence in the results of this quantal analysis. However, it was apparent that the altered shape of the histogram obtained posttetanus could not be explained by increases in quantal size alone.

\section{Paired-pulse facilitation}

Unitary EPSPs were tested for paired-pulse facilitation at an interpulse interval of $25 \mathrm{msec}$. Seven of the EPSPs showed facilitation of the mean peak amplitude of the second EPSP (ranging from 14 to $160 \%$ ); one showed a minimal change (3\%) and 2 were depressed $(-20$ and $-63 \%)$. The 3 examples of averaged EPSPs in Figure 8 indicate the variability in the degree of facilitation observed for different unitary EPSPs. The average facilitation was $35 \%(\mathrm{SD}=58 \%, n=10)$ at $25 \mathrm{msec}$ interpulse 
interval. Facilitation measurements over a range of interpulse intervals were incomplete.

\section{Discussion}

\section{Conclusions}

The major finding of this study is that LTP is rarely demonstrated in CA 1 neurons when only a single or a few CA 3 neurons are activated. Potentiation does occur readily, however, when a sufficiently large number of afferents are activated. There are 4 possibilities that occur to us to explain the low incidence of LTP expression for unitary and small compound EPSPs: (1) limitations of the experimental protocol, (2) confinement of the mechanism for potentiation to a limited number of CA3 cells, (3) the establishment or unmasking of new functional synaptic connections during LTP, and (4) a threshold mechanism for the expression of LTP. Each of these possibilities is considered in ascending order with respect to their likelihood. A threshold model is presented that confers the ability of a single CA 1 neuron to selectively potentiate its synaptic response to a variety of temporal conjunctions for different spatial combinations of inputs that share some common postsynaptic elements.

\section{Possible limitations of experimental protocol}

Although the amplitude of the small compound and unitary EPSPs generally appeared to remain stationary after tetanization, enhancement may have occurred, but by a proportion too small to be seen above the minute-by-minute variations in mean amplitude. A given percentage increment in amplitude would be more obvious for the larger compound EPSPs, which had a higher signal-to-noise ratio. However, when the mean amplitudes of the EPSPs in the nonenhanced category were considered together, no consistent trend, as distinct from individual variability, was detected in the averaged values. This argues against small (but frequently occurring) potentiations having gone unnoticed. Moreover, the occasional small compound and unitary EPSPs which were potentiated could not account for the magnitude of the changes observed in the large compound EPSPs (assuming the large EPSPs result from linear summation of unitary EPSPs). The averaged mean amplitudes (before and after the tetani) of the large compound EPSPs increased from 1.89 to $3.22 \mathrm{mV}$ ( $+63 \%$ change, $n=4)$. The small compound EPSPs averaged $200 \mu \mathrm{V}$ before the tetani and $212 \mu \mathrm{V}$ afterwards ( $+6 \%$, $n=40$ ). In the experiments where large compound and unitary EPSPs were obtained for the same CA1 neuron, the mean amplitude of the 7 of 9 compound EPSPs that were potentiated increased from 5.3 to $8.4 \mathrm{mV}(+59 \%, n=7)$, while the unitary EPSPs for this same group declined from 277 to $272 \mu \mathrm{V}(-2 \%$, $n=7$ ). Clearly, the few small EPSPs which were potentiated contributed too little to the overall averages to account for the percentage change observed in the large EPSPs.

It is unlikely that our experimental protocols were inadequate for LTP induction; indeed, LTP induction was directly demonstrated by recording the enhanced large compound EPSPs. The conjunction of presynaptic activation and postsynaptic depolarization (necessary conditions for LTP induction; see Kelso et al., 1986; Malinow and Miller, 1986; Gustafsson and Wigstrom, 1988) was obtained in our experimental protocols. In the experiments with small compound EPSPs, the same tetanization parameters induced LTP in the large compound EPSPs. These experiments employed the protocol of Gustafsson et al. (1987) where the EPSPs were conjoined with depolarizing pulses. In the unitary EPSP experiments, the compound EPSP was usually
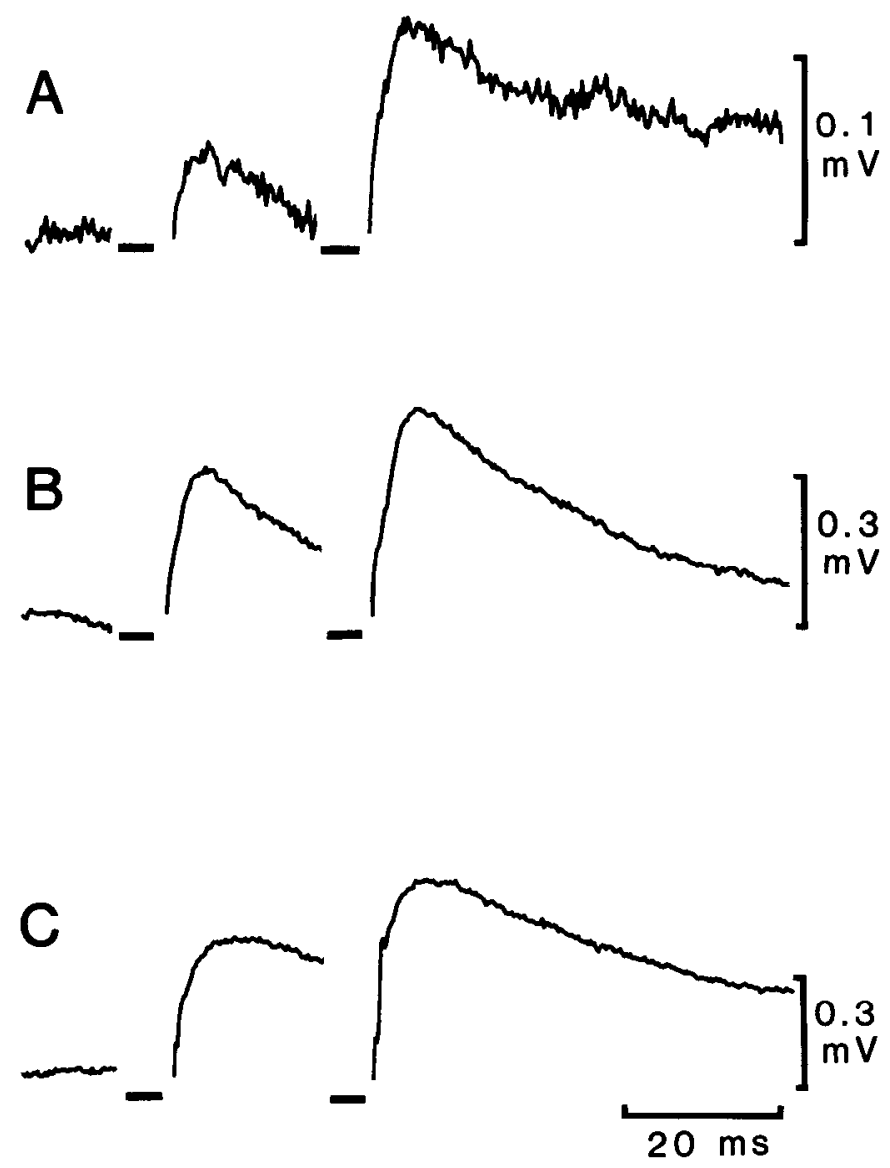

Figure 8. Three examples of averaged unitary EPSPs during paired pulse facilitation tests. The 3 examples illustrated were tested at $25 \mathrm{msec}$ interpulse intervals at a $1 \mathrm{sec}$ cycle. The degree of interaction of the test unitary EPSP with the conditioning unitary EPSP was variable among cells, as indicated by the examples illustrated. The degree of facilitation was marked for some connections $(160 \%$ in $A)$ and absent for others (3\% in $B)$; and in some cases, depression was seen $(-20 \%$ in $C)$.

potentiated, indicating LTP induction had occurred for the CA1 cell in question. Moreover, the test stimulation rate $(2 \mathrm{~Hz})$ did not preclude induction of LTP as measured by extracellular field potentials and conventional intracellular compound EPSPs (see Fig. 3).

It is possible, although unlikely, that the sites of the synaptic contacts for the unitary and small compound EPSPs were always electrotonically isolated from all of the other synaptic sites for the group of axons activated during the tetani of the SR. The evidence against this argument is multifold. First, the tetanic stimulus strength was above threshold for antidromic activation of the CA3 neurons in 4 of the 7 CA3-CA1 pairs for which the compound EPSP was potentiated while the unitary EPSP did not change (Table 1). The axon (or at least a branch of that axon) making the connection between the CA3 and the CA1 neuron was therefore among the group of afferents providing the tetanic depolarization. While this axon may have made contact with the CAl cell via a branch which deviated from the rest of the stimulated axons to a remote region of the dendritic tree, it would be no more likely to do this than any of the other activated axons. For such an axon to synapse at a region of dendrite less depolarized than other regions should therefore be an exception rather than the rule. Three of the 7 pairs had higher 
thresholds for antidromic activation of the CA3 cell, and so were tetanized using intracellular current pulses in the CA3 neuron. In the worst case, the single afferent might make a synaptic connection with the basal dendrites in the stratum oriens, while the potentiated compound EPSP results from tetanization of afferents that project to the apical dendrites. However, CA1 neurons are electrotonically compact (Turner and Schwartzkroin, 1980; Johnston, 1981; Turner, 1984a, b), and this would tend to minimize the problem caused by spatial separation.

The results of experiments using protocols 2 and 5 also argue strongly against electrotonic separation of inputs being the reason that LTP was rare for small compound or unitary EPSPs. The time courses of all the unitary EPSPs used in protocol 5 were such that they were judged to be generated at proximal dendritic sites. The largest normalized rise time/half-width was $0.2 / 1.14$, and using a cable model of CA1 pyramidal neurons (Turner, 1984b, Fig. 6, with $\alpha^{-1}=0.02=$ normalized time to peak of synaptic current), an EPSP with these shape indices would be generated at $0.6 \lambda$ from the soma. This location can be revised to $0.4-0.5 \lambda$ for the more appropriate $\alpha^{-1}$ of 0.033 (Turner, 1988). If the length of the apical dendrite is $1.0 \lambda$, a steady depolarization at the soma will be reduced by $30 \%$ at $0.5 \lambda$. It is therefore unlikely that the synapses generating the unitary EPSPs were electrotonically removed from the somatic depolarization. Furthermore, the small compound EPSPs would have been generated over a range of synaptic locations (Sayer et al., 1990), including some close to the soma, yet the expression of LTP was also infrequent for these EPSPs.

\section{Subpopulations of synapses that express LTP}

Prior to our experiments, hippocampal LTP had only been studied using field potentials or intracellularly recorded compound synaptic potentials. It was generally assumed (at least by us) that the enhancement of EPSPs elicited by the activation of many afferents was likely to reflect the summation of the enhancement that occurred at the many individual synaptic contacts that contributed to the compound EPSP. However, our results on unitary and small compound EPSPs demonstrate that this is not the case. One possible reason for this result is that LTP of compound synaptic potentials is due to selective potentiation at a subpopulation of synaptic contacts. For example, the compound EPSP (which may result from the summation of 50 unitary EPSPs) grows by $40 \%$ because perhaps 5 of the unitary EPSPs grew by $400 \%$ and not because of equal enhancement of all the contacts by $40 \%$. If such superpotentiating synapses exist, it is possible that they were not sampled in our experiments. However, of the $\mathbf{4 8}$ small compound and unitary EPSPs studied, the 5 that showed some sign of potentiation averaged a $65 \%$ increase in amplitude; this effect was usually short-lived, even though the compound EPSP remained potentiated. Thus, even the few signs of LTP expression for small EPSPs are not sufficient to account for the magnitude of the LTP observed for compound or field synaptic potentials.

Alternatively, superpotentiating synaptic connections may be extremely rare, requiring a considerably larger sample than the 48 EPSPs we studied to detect their occurrence. In light of the suggested associative role of LTP in the hippocampal formation (McNaughton et al., 1978; Levy and Steward, 1979; Barrionuevo and Brown, 1983; Wigstrom and Gustafsson, 1983; Sastry et al., 1986), the selection of a few synaptic contacts to perform the potentiating function would severely limit the capacity of this mechanism.

\section{Formation of new functional connections}

The establishment of stronger or more functional synaptic connections on dendritic spines has been proposed as a mechanism for LTP expression (Fifkova and van Harreveld, 1977; Desmond and Levy, 1983, 1986). The calcium activation of calpain (Seubert et al., 1987) and ultimately the degradation of brain spectrin has been suggested to alter the neuronal cytoskeleton affecting dendritic spine morphology. This may cause the formation of new synaptic contacts. These contacts may be additional to those which already existed between previously connected CA3-CA1 cell pairs, or they may be between previously unconnected cell pairs. In either case, when LTP is evaluated by conventional electrophysiological methods (field potentials or large compound EPSPs), the test stimulus applied to the SR would result in a larger potential after LTP induction since more synaptic sites would be activated from stimulating the same number of axons. Indeed, the results of recent ultrastructural analysis (Andersen, 1989) suggest that additional contacts are formed within $30 \mathrm{~min}$ of LTP induction. Howcver, without a complete serial reconstruction of the boutons and axons of origin to the spines and nearby dendritic shafts, it is not yet possible to discriminate between additional and de novo synaptic contacts.

In our experiments on unitary EPSPs, additional but not de novo contacts should manifest as enhanced synaptic transmission. In order to reveal the establishment of de novo connections, tetanization of the SR would have to be applied while recording from unconnected CA3-CA1 cell pairs. However, the experiments on small compound EPSPs should demonstrate either type of amplification of synaptic transmission. Several (probably 2-5; see Sayer et al., 1989, 1990) Schaffer collaterals that make functional contacts on the CA1 neuron were activated with this protocol; the same stimulus was likely to also activate some axons that did not innervate the CAl neuron. Thus, the formation of new connections from either group of afferents would be expected to result in enhancement of some small compound EPSPs. The establishment of new functional connections could also result from an unmasking of synaptic connections that are already anatomically present but did not release transmitter prior to the tetanus. However, the same arguments made above for the possible effects of new connections can be applied to this.

\section{Threshold for LTP expression}

While the mechanisms underlying induction and maintenance of LTP are often considered separately (Gustafsson and Wigstrom, 1988), it is tacitly assumed that if induction has occurred, the effects of LTP will be expressed. However, our results suggest that LTP expression should also be considered separately. The various experimental protocols that we used were sufficient to induce LTP as conventionally evaluated (by extracellular recording of field potentials or intracellular recording of large compound synaptic potentials). Moreover, our results demonstrate that the individual presynaptic CA3 neurons tested participated in the LTP induction. Although the changes that underlie LTP have occurred at the tetanized synapses, a threshold must be reached (by activating a sufficient number of afferents) during subsequent testing of the pathway for cxpression of enhanced transmission. A schematic illustration of such a thresh- 
old mechanism for the expression of LTP based on our results appears in Figure 9. The threshold may take the form of a critical level of depolarization at the postsynaptic site of the CA1 neuron or a critical amount of substance released by neighboring presynaptic terminals.

$\Lambda$ voltage threshold (cooperativity; see Bliss and GardnerMedwin, 1973; McNaughton et al., 1978) for LTP induction (vis-à-vis the relief of the magnesium block of the NMDA channel; see Mayer et al., 1984; Nowak et al., 1984) has been demonstrated. However, once LTP induction has occurred, enhanced transmission is via the quisqualate/kainate receptors, not the NMDA channel (Kauer et al., 1988; Davies et al., 1989). Thus, another candidate for mediating a thrcshold for LTP expression must be found. A shift towards more negative potentials in the current-voltage relation for a membrane channel that contributes ionic current during LTP expression would fill this role. Connor et al. (1988) proposed that a modification of voltage-dependent channels (possibly calcium channels) occurs in the dendrites of dissociated CAl neurons after application of glutamate or NMDA and that such a mechanism could occur during LTP. If a major component of LTP is a modification of voltage-dependent postsynaptic currents (by enhancing inward or reducing outward currents) in a localized region of the CA1 neuron close to the activated synapses, activation of only one or a few CA3 afferents may be insufficient for the expression of LTP. The appropriate channel modification occurs after the tetanus (induction), but unless the channels are exposed to a level of depolarization past their activation threshold by subsequent test inputs, expression does not occur.

In support of the hypothesis for a voltage threshold for the expression of LTP, the single case of unitary EPSP enhancement (Fig. 7) occurred when the normalized control amplitude was one of the largest we observed. The mean peak amplitude of this EPSP was $300 \mu \mathrm{V}$ and the normalized rise time was 0.20 . These values are plotted in Figure $5 C$ of Sayer et al. (1990). Assuming that common rise times indicate similar electrotonic distances between the location of the spine(s) involved and the soma, this suggests that even before the tetanization, this EPSP was one of the largest at its dendritic origin. Moreover, a recent report (Miles, 1988) indicates that similar experiments on the considerably larger amplitude unitary EPSPs between CA3-CA3 cell pairs may be more effective at expressing LTP.

\section{Functional implications for a threshold model for LTP expression}

A threshold mechanism for the expression of LTP would reduce ambiguities in CAl discharge that could result subsequent to LTP induction at synapses arising from a group of afferents. Such ambiguities could result from amplification of the noise when a single afferent or a small subgroup of the original association of tetanized afferents was activated. Without a threshold, their potentiatcd responses could override the effects of other weaker or nonassociative signals and lead to a situation where potentiation of uncorrelated activity in individual components of the original association mask the postsynaptic responses of other inputs to the CAl neuron. A threshold mechanism also offers the advantage of specificity to the synapses that participated in the tetanization through electrotonic isolation and/or local modifications to the effectiveness of synaptic transmission. Another advantage of a threshold mechanism is that an individual synapse can participate in multiple associations (see Fig. 9 legend). Thus, the combinatorial capacity of
A

CA3

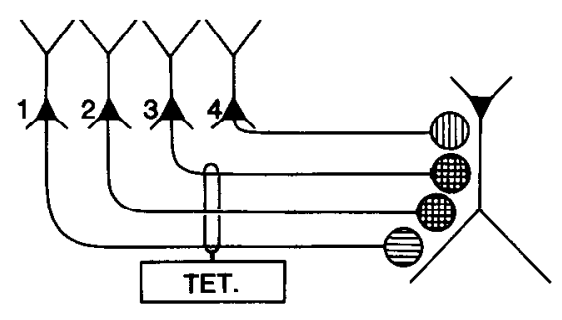

B
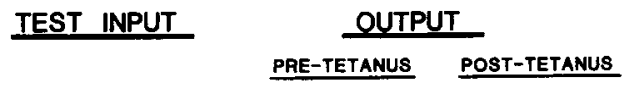

ANY ONE CA3

ANY TWO CA3:

$1,2 \& 3$

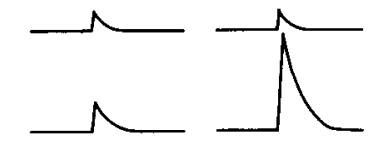

$2,3 \& 4$

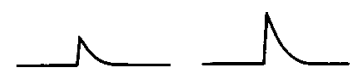

Figure 9. Schematic representation of results suggesting a threshold for LTP expression. A, Diagram of a CA1 neuron receiving convergent monosynaptic inputs from many (4 are illustrated for clarity) $\mathrm{CA} 3$ neurons. The presynaptic terminals of each $\mathrm{CA} 3$ neuron are indicated by enclosed lines with respect to several associations they may participate in. Inputs 1, 2, and 3 form onc association; 2, 3, and 4 form another association ( 2 and 3 are common elements to both associations). The $1-2-3$ association is the one tetanized for purposes of this schematic diagram. $B$, The test input (which $\mathrm{CA} 3$ neurons are simultaneously activated) for a variety of spatial combinations is illustrated with the evoked EPSPs before and after tetanization of the 1,2 , and 3 CA3 inputs. This arrangement provides for the specificity of the potentiated response to the appropriate synapses. Several possible mechanisms could contribute to the specificity, including electrotonic isolation of potentiated inputs or local modification of postsynaptic membrane receptors and/or channels, presynaptic terminals, or both.

the CA3-CA1 pathway for associative interactions is greatly increased.

\section{References}

Abraham, W. C., B. Gustafsson, and H. Wigstrom (1987) Long-term potentiation involves enhanced synaptic excitation relative to synaptic inhibition in the guinea-pig hippocampus. J. Physiol. (Lond.) 394: 67-380.

Andersen, P. (1989) Cellular and molecular mechanisms for LTP induction and maintenance. Proceedings of the 1989 Miami Biotechnology Winter Symposium, Vol. 9, pp. 90-91.

Andersen, P., S. H. Sundberg, O. Sveen, J. W. Swann, and H. Wigstrom (1980) Possible mechanisms for long-lasting potentiation of synaptic transmission in hippocampal slices from guinea-pigs. J. Physiol. (Lond.) 302: 463-482.

Artola, A., and W. Singer (1987) Long-term potentiation and NMDA receptors in rat visual cortex. Nature 330: 649-652.

Barrionuevo, G., and T. H. Brown (1983) Associative long-term potentiation in hippocampal slices. Proc. Natl. Acad. Sci. USA 80: 73477351.

Baudry, M., M. Oliver, R. Creager, A. Wieraszko, and G. Lynch (1980) Increase in glutamate receptors following repetitive electrical stimulation in hippocampal slices. Life Sci. 27: 325-330.

Bliss, T. V. P., and A. R. Gardner-Medwin (1973) Long-lasting potentiation of synaptic transmission in the dentate area of the unanaesthetised rabbit following stimulation of the perforant path. J. Physiol. (Lond.) 232: 357-374. 
Bliss, T. V. P., and T. Lomo (1973) Long-lasting potentiation of synaptic transmission in the dentate area of the anaesthetised rabbit following stimulation of the perforant path. J. Physiol. (Lond.) 232: 331-356.

Bliss, T. V. P., R. M. Douglas, M. L. Errington, and M. A. Lynch (1986) Correlation between long-term potentiation and release of endogenous amino acids from dentate gyrus of anaesthetized rats. J. Physiol. (Lond.) 377: 391-408.

Brown, T. H., and D. A. McAfee (1982) Long-term synaptic potentiation in the superior cervical ganglion. Science 215: 1411-1413.

Chang, F.-L. F., and W. T. Greenough (1984) Transient and enduring morphological correlates of synaptic activity and efficacy change in the rat hippocampal slice. Brain Res. 309: 35-46.

Collingridge, G. L., S. J. Kehl, and H. McLennan (1983) The antagonism of amino acid-induced excitations of rat hippocampal CAl neurones in vitro. J. Physiol. (Lond.) 334: 19-31.

Connor, J. A., W. J. Wadman, P. E. Hockberger, and R. K. S. Wong (1988) Sustained dendritic gradients of $\mathrm{Ca}^{2+}$ induced by excitatory amino acids in CA1 hippocampal neurons. Science 240: 649-653.

Davies, S. N., R. A. J. Lester, K. G. Reymann, and G. L. Collingridge (1989) Temporally distinct pre- and post-synaptic mechanisms maintain long-term potentiation. Nature 338: 500-503.

Desmond, N. L., and W. B. Levy (1983) Synaptic associative potentiation/depression. An ultrastructural study in the hippocampus. Brain Res. 265: 21-30.

Desmond, N. L., and W. B. Levy (1986) Changes in the numerical density of synaptic contacts with long-term potentiation in the hippocampal dentate gyrus. J. Comp. Neurol. 253: 466-475.

Dolphin, A. C., M. L. Errington, and T. V. P. Bliss (1982) Long-term potentiation of the perforant path in vivo is associated with increased glutamate release. Nature 297: 496-498.

Douglas, R. M., G. V. Goddard, and M. Riives (1982) Inhibitory modulation of long-term potentiation: Evidence for a postsynaptic locus of control. Brain Res. 240: 259-272.

Dunwiddie, T., and G. Lynch (1978) Long-term potentiation and depression of synaptic responses in the rat hippocampus: Localization and frequency dependency. J. Physiol. (Lond.) 276: 353-367.

Frrington, M. L., M. A. Lynch, and T. V. P. Bliss (1987) Long-term potentiation in the dentate gyrus: Induction and increased glutamate release are blocked by $\mathrm{D}(-)$ aminophosphonovalerate. Neuroscience 20: 279-284.

Fifkova, E., and A. van Harreveld (1977) Long-lasting morphological changes in dendritic spines of dentate granule cells following stimulation of entorhinal area. J. Neurocytol. 6: 211-230.

Gerren, R. A., and N. M. Weinberger (1983) Long-term potentiation in the magnocellular medial geniculate nucleus of the anesthetized cat. Brain Res. 265: 138-142.

Gustafsson, B., and H. Wigstrom (1986) Hippocampal long-lasting potentiation produced by pairing single volleys and brief conditioning tetani evoked in separate afferents. J. Neurosci. 6: 1575-1582.

Gustafsson, B., and H. Wigstrom (1988) Physiological mechanisms underlying long-term potentiation. Trends Neurosci. 11: 156-162.

Gustafsson, B., H. Wigstrom, W. C. Abraham, and Y.-Y. Huang (1987) Long-term potentiation in the hippocampus using depolarizing current pulses as the conditioning stimulus to single volley synaptic potentials. J. Neurosci. 7: 774-780.

Haas, H. L., and G. Rose (1984) The role of inhibitory mechanisms in hippocampal long-term potentiation. Neurosci. Lett. 47: 301-306.

Harris, E. W., A. H. Ganong, and C. W. Cotman (1984) Long-term potentiation in the hippocampus involves activation of $\mathrm{N}$-methylaspartate receptors. Brain Res. 323: 132-137.

Hinton, G. E., and J. A. Anderson, eds. (1981) Parallel Models of Associative Memory, Lawrence Erlbaum, Hillsdale, NJ.

Johnston, D. (1981) Passive cable properties of hippocampal CA3 pyramidal neurons. Cell. Mol. Neurobiol. 1: 41-55.

Kauer, J. A., R. C. Malenka, and R. A. Nicoll (1988) A persistent postsynaptic modification mediates long-term potentiation in the hippocampus. Neuron 1: 911-917.

Kelso, S. R., A. H. Ganong, and T. H. Brown (1986) Hebbian synapses in hippocampus. Proc. Natl. Acad. Sci. USA 83: 5326-5330.

Larson, J., and G. Lynch (1986) Induction of synaptic potentiation in hippocampus by patterned stimulation involves two events. Science 232: 985-988.

Lee, K.S. (1982) Sustained enhancement of evoked potentials following brief, high frequency stimulation of the cerebral cortex, in vitro. Brain Res. 239: 617-623.
Lee, K. S., F. Schottler, M. Oliver, and G. Lynch (1980) Brief bursts of high-frequency stimulation produce two types of structural change in rat hippocampus. J. Neurophysiol. 44: 247-258.

Levy, W. B., and O. Steward (1979) Synapses as associative memory elements in the hippocampal formation. Brain Res. 175: 233-245.

Lynch, G., and M. Baudry (1984) The biochemistry of memory: A new and specific hypothesis. Science 224: 1057-1063.

Lynch, M. A., and T. V. P. Bliss (1986) On the mechanism of enhanced release of $\left[{ }^{14} \mathrm{C}\right]$ glutamate in hippocampal long-term potentiation. Brain Res. 369: 405-408.

Lynch, G., V. K. Gribkoff, and S. A. Deadwyler (1976) Long-term potentiation is accompanied by a reduction in dendritic responsiveness to glutamic acid. Nature 263: 151-153.

Lynch, G., S. Halpain, and M. Baudry (1982) Effects of high-frequency synaptic stimulation on glutamate receptor binding studied with a modified in vitro hippocampal slice preparation. Brain Res. 244: 101111 .

MacDermott, A. B., M. L. Mayer, G. L. Westbrook, S. J. Smith, and J. L. Barker (1986) NMDA-receptor activation increases cytoplasmic calcium concentration in cultured spinal cord neurones. Nature 321: 519-522.

Malinow, R., and J. P. Miller (1986) Postsynaptic hyperpolarization during conditioning reversibly blocks induction of long-term potentiation. Nature $320: 529-530$

Mayer, M. L., G. L. Westbrook, and P. B. Guthrie (1984) Voltage dependent block by $\mathrm{Mg}^{++}$of NMDA responses in spinal cord neurones. Nature 309: 261-263.

McNaughton, B. L., R. M. Douglas, and G. V. Goddard (1978) Synaptic enhancement in fascia dentata: Cooperativity among coactive afferents. Brain Res. 157: 277-293.

Miles, R. (1988) Plasticity of recurrent excitatory synapses between CA3 hippocampal pyramidal cells. Soc. Neurosci. Abstr. 14: 19.

Nowak, L., P. Bregestovski, P. Ascher, A. Herbert, and A. Prochiantz (1984) Magnesium gates glutamate-activated channels in mouse central neurones. Nature 307: 462-465.

Robinson, G. B. (1986) Enhanced long-term potentiation induced in rat dentate gyrus by coactivation of septal and entochinal inputs: Temporal constraints. Brain Res. 379: 56-62

Sastry, B. R., J. W. Goh, and A. Auyeung (1986) Associative induction of posttetanic and long-term potentiation in CAl neurons of rat hippocampus. Science 232: 988-990.

Sayer, R. J., M. J. Friedlander, and S. J. Redman (1988) Long-term potentiation compared for unitary and compound EPSPs in CAl neurones. Soc. Neurosci. Abstr. 14: 19.

Sayer, R. J., S. J. Redman, and P. Andersen (1989) Amplitude fluctuations in small EPSPs recorded from CA1 pyramidal cells in the guinea-pig hippocampal slice. J. Neurosci. 9: 840-850.

Sayer, R. J., M. J. Friedlander, and S. J. Redman (1990) The time course and amplitude pairs of EPSPs evoked at synapses between pairs of CA3/CA1 neurons in the hippocampal slice. J. Neurosci. 10 . 826-836.

Seubert, P. M., M. Baudry, S. Dudek, and G. Lynch (1987) Calmodulin stimulates the degradation of brain spectrin by calpain. Synapse 1 20-24.

Skrede, K. K., and D. Malthe-Sorenssen (1981) Increased resting of an evoked release of transmitter following repetitive electrical tetanization in hippocampus: A biochemical correlate to long-lasting synaptic potentiation. Brain Res. 208: 436-441.

Smith, S. J. (1987) Progress on LTP at hippocampal synapses: A postsynaptic $\mathrm{Ca}^{2+}$ trigger for memory storage? Trends Neurosci. 10: 142144.

Taube, J. S., and P. A. Schwartzkroin (1987) Intracellular recording from hippocampal CAl interneurons before and after development of long-term potentiation. Brain Res. 419: 32-38.

Taube, J. S., and P. A. Schwartzkroin (1988) Mechanisms of longterm potentiation: EPSP/spike dissociation, intradendritic recordings, and glutamate sensitivity. J. Neurosci. 8: 1632-1644.

Teyler, T. J., and P. DiScenna (1984) Long-term potentiation as a candidate mnemonic device. Brain Res. Rep. 7: 15-28.

Teyler, T. J., and P. DiScenna (1987) Long-term potentiation. Annu. Rev. Neurosci. 10: 131-161.

Turner, D. A. (1984a) Segmental cable evaluation of somatic transients in hippocampal neurons (CA1, CA3 and dentate). Biophys. J. 46: 7384.

Turner, D. A. (1984b) Conductance transients onto dendritic spines 
in a segmental cable model of hippocampal neurons. Biophys. J. 46: $85-96$.

Turner, D. A. (1988) Waveform and amplitude characteristics of evoked responses to dendritic stimulation of CAl guinea-pig pyramidal cells. J. Physiol. (Lond.) 395: 419-439.

Turner, D. A., and P. A. Schwartzkroin (1980) Steady-state electrotonic analysis of intracellularly stained hippocampal neurons. J. Neurophysiol. 44: 184-199.

Wigstrom, H., and B. Gustafsson (1985) Facilitation of hippocampal long-lasting potentiation by GABA antagonists. Acta Physiol. Scand. 125: $159-172$.
Wigstrom, H., B. Gustafsson, Y.-Y. Huang, and W. C. Abraham (1986a) Hippocampal long-term potentiation is induced by pairing single afferent volleys with intracellularly injected depolarizing current probes. Acta Physiol. Scand. 126: 317-319.

Wigstrom, H., B. Gustafsson, and Y.-Y. Huang (1986b) Mode of action of excitatory amino acid receptor antagonists on hippocampal long-lasting potentiation. Neuroscience 17: 1105-1115.

Yamamoto, C., and S. Sawada (1981) Important factors in the induction of long-term potentiation in thin hippocampal slices. Exp. Ncurol. 74: 122-130. 